\title{
Acute Vascular Effects of Aquatic and Land Treadmill Exercise
}

Dustin P. Joubert ${ }^{1}$, Peter W. Grandjean ${ }^{2}$, and Stephen F. Crouse ${ }^{1}$

${ }^{1}$ Applied Exercise Science Laboratory; Department of Health \& Kinesiology; Texas A\&M University; College Station, TX ${ }^{2}$ Baylor Laboratories for Exercise Science \& Technology; Department of Health, Human Performance, Recreation; Baylor University; Waco, TX

Category: Doctoral

Advisor/Mentor: Crouse, Stephen F. (crouse@hlkn.tamu.edu)

ABSTRACT

Aquatic treadmill (ATM) training has been shown to reduce blood pressure reactivity to exercise to a greater extent than land treadmill (LTM) training in an older, overweight, and sedentary population (Greene, MSSE 2013). Less is known regarding the mechanisms or the acute effects. Purpose: Determine the acute vascular effects of ATM and LTM exercise in healthy, recreationally active college males. Methods: Seven male subjects ( $21 \pm 1$ years; $175 \pm 8 \mathrm{~cm} ; 73.7 \pm 9.5 \mathrm{~kg} ; 24 \pm 3 \mathrm{~kg} / \mathrm{m}^{2}$ ) performed two, 30 minute acute exercise bouts (ATM and LTM) at $\sim 75 \%$ MHR in a randomized fashion, matched for VO2 (ATM: $33.3 \pm 6.1$, LTM: $34.7 \pm 6.0 \mathrm{ml} \bullet \mathrm{kg}^{-1} \bullet \mathrm{min}^{-1}$ ). Flow mediated dilation (FMD) and carotid-femoral pulse wave velocity (PWV) were recorded by ultrasound using standard procedures prior to and two hours after each session. Blood pressure was recorded following supine rest before exercise and averaged from post exercise recordings taken every 15 minutes from 30-120 minutes post. Changes in each variable were calculated pre and post exercise for each mode and compared between modes by a dependent sample $t$ test.

Results:

\begin{tabular}{cccccccc}
\hline & & FMD & PWV & SBP & DBP & MAP & HR \\
\hline \multirow{4}{*}{ ATM } & Pre-Ex & $9.5 \pm 4.2$ & $7.1 \pm 1.7$ & $116 \pm 7$ & $62 \pm 7$ & $80 \pm 7$ & $59 \pm 10$ \\
& Post-Ex & $6.5 \pm 1.7$ & $7.6 \pm 2.0$ & $113 \pm 9$ & $62 \pm 7$ & $79 \pm 7$ & $61 \pm 6$ \\
& Change & $-3.0 \pm 4.6$ & $0.6 \pm 1.0$ & $-2.9 \pm 5.2$ & $0.1 \pm 1.1$ & $-0.9 \pm 2.0$ & $2.3 \pm 6.9$ \\
\hline \multirow{3}{*}{ LTM } & Pre-Ex & $6.4 \pm 2.7$ & $7.1 \pm 0.8$ & $116 \pm 8$ & $62 \pm 7$ & $80 \pm 7$ & $58 \pm 8$ \\
& Post-Ex & $7.1 \pm 3.3$ & $6.5 \pm 1.1$ & $116 \pm 7$ & $60 \pm 8$ & $79 \pm 8$ & $62 \pm 8$ \\
& Change & $0.7 \pm 4.2$ & $-0.6 \pm 0.8$ & $-0.3 \pm 7.4$ & $-1.9 \pm 4.6$ & $-1.4 \pm 4.9$ & $3.7 \pm 4.3$ \\
\hline & P-Value & 0.237 & 0.078 & 0.581 & 0.239 & 0.821 & 0.535 \\
\hline
\end{tabular}

Values represent mean $\pm \mathrm{SD}$; P-Value from dependent sample T-Test on Change variables.

FMD (\% change from baseline); PWV (m/s).

Conclusion: No significant changes found pre and post exercise between modes. While exercise modes were matched for VO2, RPE was higher for ATM $(14.2 \pm 1.7)$ than LTM $(12.7 \pm 1.5)$, potentially indicating varying degrees of sympathetic stimulation. While this could potentially explain the slight reduction in FMD, in the present population of young, recreationally active men studied, no differences can be substantiated between ATM and LTM. Lack of significant findings may have also been due to the small sample size and reduced statistical power. Future research should focus on less active, older populations in response to ATM and LTM exercise. 